\title{
PA-136 EFFECT OF ONCHOCERCIASIS TREATMENT ON THE FREQUENCY OF SEIZURES IN PATIENTS WITH EPILEPSY AND ONCHOCERCIASIS
}

Mandro Michel. Provincial Division of Health of Ituri, Republic of Congo

\subsection{6/bmjgh-2016-000260.162}

Background A high prevalence of epilepsy is mainly observed in Onchocerca volvulus (OV) hyperendemic areas with no or low ivermectin coverage. There is anecdotal evidence that ivermectin may reduce seizure frequency in patients with onchocerciasis associated epilepsy (OAE).

Methods Between 2008 and 2012, Rethy in Ituri Province, Democratic Republic of Congo, was a study site for a phase III trial comparing moxidectin versus ivermectin as treatment for subjects infested with OV. Participants received a single oral dose of OV drug and were followed for 18 months. Parasitological efficacy was assessed by skin snip exams. In July 2016, the randomisation code has not been broken yet. In 2015 we traced 7 families of patients with epilepsy who had been enrolled in the trial. We interviewed them and reviewed the trial case report forms. 
Results Of 472 trial participants, $13(2.7 \%)$ had a medical history of active convulsive epilepsy. After OV treatment, 6 $(80 \%)$ of 7 male patients with epilepsy became seizure free during the following 18 months. Seizures continued in this period in only 1 person with a decrease in frequency; in the latter microfilariae remained detectable in the skin. In all subjects who became seizure free, the skin snips too became microfilaria free for at least 6 months. None of the patients received any anti-epileptic drug nor an additional dose of moxidectin or ivermectin during or after the trial. In all subjects the frequency of seizures increased again after the 18 months and 2 patients died in 2015, because of drowning in the river during seizures.

Conclusions This study suggests that moxidectin and/or ivermectin may be able to decrease the frequency of seizures in OV-infested people with epilepsy. A clinical trial will be needed to support this hypothesis. 